KODAI MATH. SEM. REP.

23 (1971), 267-275

\title{
EXTREMAL PROPERTIES OF QUASIHARMONIC FORMS AND FUNCTIONS
}

\author{
By Kazumitsu Kawai and Leo Sario
}

The purpose of the present paper is to deduce extremal properties of differential forms $\varphi$ satisfying the differential equations

$$
\delta d T \varphi+P T \varphi=0
$$

or

$$
\delta d T \varphi+d \delta S \varphi=0
$$

on a Riemannian space. For suitable choices of the operators $T$ and $S$ and the nonnegative function $P$ we obtain, in a unified manner, extremal properties of harmonic, semiharmonic, cosemiharmonic, quasiharmonic, and coquasiharmonic forms, and harmonic, $P$-harmonic, quasiharmonic, and $P$-quasiharmonic functions.

\section{$\S 1$. Fundamentals.}

1. Let $F(u, v)$ be a bilinear form on a real linear space $V$, and set $F(u)=F(u, u)$. Consider a subset $H$ of $V$ such that for each $h \in H, F(h) \geqq 0$. For a fixed $u \in V$ set $v=u+h$ for $h \in H$. We characterize $u$ by an extremal property.

The function $u$ minimizes the functional $\{F(v)-F(h, u)-F(u, h)\}$ and the minumum is $F(u)$ :

$$
F(v)-F(h, u)-F(u, h)=F(u)+F(h) .
$$

If $F$ is an inner product, set

$$
F(u, v)=[u, v], \quad \mid\left\|u^{2}\right\|=[u, u] .
$$

The function $u$ minimizes the functional $\left\{\|v \mid\|^{2}-2[h, u]\right\}$ among all $v \in V$ with $h=v-u$, and the minimum is $\|u\|^{2}$ :

$$
\left\||| v||^{2}-2[h, u]=\left|\left\|u\left|\|^{2}+\right||| h||^{2} .\right.\right.\right.
$$

We specialize further.

Received October 5, 1970.

This work was sponsored by the U.S. Army Research Office-Durham, Grant DA-AROD-31-124-70-G7, Unıversity of California, Los Angeles. 
The function $u$ minimizes $\|v\| \|$ among all $v=u+h$ with $[h, u]=0$ :

$$
\left\|\left|\|\|^{2}=\|u \mid\|^{2}+\|h\|^{2} .\right.\right.
$$

We shall make use of these simple formulas in our unifying treatment of certain minimum problems for functions and forms.

2. Let $R$ be a Riemannian space of dimension $n$. If $d$ is the exterior differential operator, and $*$ the Hodge star operator on the algebra of differential forms on $R$, then the codifferential operator $\delta$ is defined by $\delta \varphi=(-1)^{n p+n+1} * d * \varphi$ where $\varphi$ is a $p$ form. These operations satisfy

$$
\begin{gathered}
d d \varphi \equiv 0, \quad * * \varphi=(-1)^{n p+p} \varphi, \quad \delta \delta \varphi \equiv 0, \\
* \delta d \varphi=d \delta * \varphi, \quad \delta d * \varphi=* d \delta \varphi .
\end{gathered}
$$

We consider a compact subregion $\Omega$ with smooth boundary surface $\partial \Omega$ and assume that all forms are sufficiently smooth on $\partial \Omega$. An inner product and norm on the space of $p$-forms are given by

$$
(\alpha, \beta)=\int_{\Omega} \alpha \wedge * \beta, \quad\|\alpha\|^{2}=(\alpha, \alpha) .
$$

We shall make use of Stokes' formula for $p$-forms $\varphi$ :

$$
\int_{c} d \varphi=\int_{\partial c} \varphi
$$

where $c$ is a chain of dimension $p+1$ and $\partial c$ its boundary. From this follows Green's formula

$$
(d \varphi, \phi)-(\varphi, \delta \psi)=\int_{\partial \Omega} \varphi \wedge * \psi
$$

for $(p-1)$-forms $\varphi$ and $p$-forms $\phi$.

We denote by t $\varphi$ and $n \varphi$ the tangential and normal components of $\varphi$ on $\partial \Omega$ and recall that

$$
* t=n *, \quad * n=t * .
$$

We shall be dealing with a linear operator $T$ which maps the exterior algebra of differential forms into itself. The operators $d, *$, and $\delta$ are examples of such a $T$. In the sequel we mainly consider as $T$ various combinations of these three operators.

Let $P$ be a $C^{\infty}$-function defined on $\Omega$ with $P \geqq 0$, and consider the differential equation for $p$-forms:

$$
\delta d T \varphi+P T \varphi=0
$$


Our result will be valid under much less stringent conditions on $P$, but this aspect of the problem is not a topic of this paper. We associate with (9) a generalized Dirichlet integral

$$
[\varphi, \phi]=(d T \varphi, d T \psi)+(P T \varphi, T \psi),
$$

which is an inner product for $p$-forms $\varphi, \phi$. Our aim is to show that a solution of (9) is extremal in the minimum problem concerning the norm $\|\mid \psi\| \|^{2}=[\psi, \phi]$ induced by the above integral, and that the basic formulas in 1 serve as a simple unifying device in the study of extremal properties of various forms and functions.

3. We begin with the following preliminary result.

Proposition 1. If a p-form $\varphi$ satisfies (9), then among p-forms $\phi$ with $t T \psi=t T \varphi$ on $\partial \Omega, \varphi$ minimizes the functional $\|\phi\|\left\|^{2}=\right\| d T \psi \|^{2}+(P T \psi, T \psi)$. Explicitly,

$$
\||\phi|\|^{2}=\left\|\left|\varphi\left\|^{2}+\right\|\right| \eta\right\|^{2}, \quad \text { where } \eta=\phi-\varphi \text {. }
$$

Proof. By (7) and (9),

$$
\begin{aligned}
{[\eta, \varphi] } & =(d T \eta, d T \varphi)+(P T \eta, T \varphi) \\
& =(d T \eta, d T \varphi)-(T \eta, \delta d T \varphi) \\
& =\int_{\partial \Omega} T \eta \wedge * d T \varphi .
\end{aligned}
$$

The condition $t T \eta=t T \psi-t T \varphi=0$ on $\partial \Omega$ leads to $[\eta, \varphi]=0$, and formula (4) gives the result.

Let $E$ be the space of $p$-forms $\varphi$ on $\Omega$ with $\|\varphi\| \|<\infty$. Denote by $E_{P, T}$ the subspace of $E$ consisting of solutions of (9), and by $E_{0}$ the subspace consisting of those $\varphi$ for which $t T \varphi=0$. If $T$ and $P$ are so chosen that equation (9) has a unique solution $\varphi$, with given boundary values $t T \varphi$. Then we have:

Proposition 2. The following orthogonal decomposition is valid:

$$
E=E_{P, T}+E_{0} .
$$

4. We illustrate the above statement by discussing special cases of the operator $T$.

We consider the Laplace-Beltrami operator

$$
\Delta=\delta d+d \delta
$$

and the simpler operators

$$
\Delta^{\prime}=\delta d, \quad \Delta^{\prime \prime}=d \delta .
$$

First we take the identity operator as $T$. Then equation (9) simplifies to 
$\delta d \varphi+P \varphi=0$, that is, $\Delta^{\prime} \varphi+P \varphi=0$. By Proposition 1, we have immediately:

Theorem 1. If $\varphi$ satisfies $\Delta^{\prime} \varphi+P \varphi=0$, then among those $\phi$ for which $t \phi=t \varphi$ on $\partial \Omega, \varphi$ gives the minimum of $\left\{\|d \phi\|^{2}+(P \psi, \phi)\right\}$.

For $T=*$, (9) is $d \delta \varphi+P \varphi=0$, that is, $\Delta^{\prime \prime} \varphi+P \varphi=0$. In fact, $\delta d * \varphi+P * \varphi=0$ reduces to $* d \delta \varphi+* P \varphi=0$ by (6). Hence $d \delta \varphi+P \varphi=0$.

The condition $t * \psi=t * \varphi$ on $\partial \Omega$ is equivalent to $* n \psi=* n \varphi$, that is, $n \psi=n \varphi$ by (8). Moreover, $\|d * \phi\|^{2}=\|\delta \phi\|^{2}$. Thus we may state:

Theorem 2. If $\varphi$ satisfies $\Delta^{\prime \prime} \varphi+P \varphi=0$, then among those $\phi$ for which $n \psi=n \varphi$ on $\partial \Omega, \varphi$ gives the minimum of $\left\{\|\delta \phi\|^{2}+(P \psi, \phi)\right\}$.

For $T=* d$, (9) is $\delta d * d \varphi+P * d \varphi=0$. By (6), we have $* d \delta d \varphi+* P d \varphi=0$. Hence $d \delta d \varphi+P d \varphi=0$. But $d^{2}=0$ implies $d \delta d=d \Delta$. Thus (9) is $d \Delta \varphi+P d \varphi=0$.

The condition $t * d \psi=t * d \varphi$ on $\partial \Omega$ becomes $* n d \psi=* n d \varphi$ by (8), i.e. $n d \psi=n d \varphi$. Moreover, $\|d * d \psi\|^{2}$ is equal to $\|\delta d \psi\|^{2}$. Therefore we obtain:

TheOREM 3. If $\varphi$ satisfies $d \Delta \varphi+P d \varphi=0$, then among those $\phi$ for which $n d \psi=n d \varphi$ on $\partial \Omega$, $\varphi$ gives the minimum of $\left\{\|\delta d \varphi\|^{2}+(P d \psi, d \psi)\right\}$.

For $T=\delta$, (9) is $\delta d \delta \varphi+P \delta \varphi=0$. Hence $\delta \Delta \varphi+P \delta \varphi=0$.

THEOREM 4. If $\varphi$ satisfies $\delta \Delta \varphi+P \delta \varphi=0$, then among those $\phi$ for which t $\delta \phi=t \delta \varphi$ on $\partial \Omega, \varphi$ gives the minimum of $\left\{\|d \delta \varphi\|^{2}+(P \delta \varphi, \delta \varphi)\right\}$.

The special case $P \equiv 0$ brings forth more interesting results. We shall study this case in the next section.

\section{§ 2. Extremal properties of harmonic and other forms.}

1. Solutions of the Laplace-Beltrami equation $\Delta \varphi=\delta d \varphi+d \delta \varphi=0$ are called harmonic forms. We also consider the weaker equation $\Delta^{\prime} \varphi=\delta d \varphi=0$ and call its solutions semiharmonic forms. In analogy, solutions of $\Delta^{\prime \prime} \varphi=d \delta \varphi=0$ will be referred to as cosemiharmonic forms.

Other classes of forms to be considered are the solutions of $d \Delta \varphi=d \delta d \varphi=0$ and those of $\delta \Delta \varphi=\delta d \delta \varphi=0$. We call such forms quasiharmonic and coquasiharmonic respectively. Note that harmonic forms are included in each of the latter two classes of forms.

We remark that $\varphi$ is cosemiharmonic (resp. coquasiharmonic) if and only if $* \varphi$ is semiharmonic (resp. quasiharmonic).

In this section we unify the treatment of harmonic, semi-harmonic, cosemiharmonic, quasiharmonic, and coquasiharmonic forms in our minimum problem by considering the equation

$$
\delta d T \varphi+d \delta S \varphi=0
$$


where $T$ and $S$ are linear operators of the exterior algebra of $p$-forms into itself, and by associating with (11) an inner product

$$
[\varphi, \psi]=(d T \varphi, d T \psi)+(\delta S \varphi, \delta S \psi) .
$$

For $p$-forms $\eta$ and $\varphi$, Green's formula yields

$$
\begin{aligned}
{[\eta, \varphi] } & =\left(d T_{\eta}, d T \varphi\right)+(\delta S \eta, \delta S \varphi) \\
& =\left(T_{\eta}, \delta d T \varphi\right)+\int_{\partial \Omega} T \eta \wedge * d T \varphi+(S \eta, d \delta S \varphi)-\int_{\partial \Omega} \delta S \varphi \wedge * S \eta \\
& =(T \eta-S \eta, \delta d T \varphi)+\int_{\partial \Omega} T \eta \wedge * d T \varphi-\delta S \varphi \wedge * S \eta
\end{aligned}
$$

For forms satisfying (11) we shall make use of the following immediate consequence of (4):

Proposition 3. Among p-forms $\phi$ with $\phi=\varphi+\eta$ and $[\eta, \varphi]=0, \varphi$ minimizes $\left\{\|d T \psi\|^{2}+\|\delta S \psi\|^{2}\right\}$.

2. Examples, I. We consider harmonic forms. Take the identity operator as $T$ and $S$. Then (11) is $\Delta \varphi=0$ and we obtain from (12),

$$
[\eta, \varphi]=\int_{\partial \Omega} \eta \wedge * d \varphi-\delta \varphi \wedge * \eta \text {. }
$$

The integral vanishes if $t \eta=0$ and $t * \eta=0$ or $n \eta=0$ on $\partial \Omega$.

In view of this and Proposition 3:

THEOREM 5. If $\varphi$ is a harmonic form, then among p-forms $\phi$ with $\phi=\varphi+\eta$ such that $t_{\eta}=n \eta=0$ on $\partial \Omega, \varphi$ gives the minimum of $\left\{\|d \psi\|^{2}+\|\delta \phi\|^{2}\right\}$.

Duff [1], [2] proved that there exists a harmonic form $\varphi$ with given boundary values of $t \varphi$ and $n \varphi$. It is known that the solution is unique if the metric tensor $g_{i}$ of the Riemannian space is analytic or if the Dirichlet problem of the equation $d \varphi=\delta \varphi=0$ has a unique solution. We call either of these the "uniqueness condition."

Using this result of Duff, we can give the above theorem the following interpretation, which can be considered as a generalization of the Dirichlet principle to forms.

COROllary 1. Among p-forms $\phi$ with given boundary values of t $\psi$ and $n \psi$, there exists a minimizing form $\varphi$ of the functional $\left\{\|d \psi\|^{2}+\|\delta \phi\|^{2}\right\}$, and $\varphi$ is a harmonic form.

If the uniqueness condition holds on $R$, then $\varphi$ is unique.

Let $E$ denote the space of forms $\phi$ with $\|d \psi\|^{2}+\|\delta \psi\|^{2}<\infty, H$ the subspace of $E$ consisting of harmonic forms, and $E_{0}$ the subspace consisting of those $\phi$ with 
vanishing $t \psi$ and $n \psi$. By Proposition 2 we have another consequence of Theorem 5:

COROLLARY 2. Under the uniqueness condition the following orthogonal decomposition is valid:

$$
E=H+E_{0},
$$

that is, for any $\phi \in E$ there exist unique $\varphi \in H$ and $\eta \in E_{0}$ such that $\phi=\varphi+\eta$ and

$$
\left\|\psi | \| ^ { 2 } = \| | \left|\varphi\left\|\left.\right|^{2}+\right\| \eta\|\|^{2}\right.\right.
$$

where

$$
\|\psi\|\left\|^{2}=\right\| d \psi\left\|^{2}+\right\| \delta \phi \|^{2}
$$

3. Examples, II. Here we consider semiharmonic forms. Take the identity operator as $T$, and set $S=0$. Then from Proposition 3 we have:

Theorem 6. Let $\varphi$ be a semiharmonic form, i.e. $\Delta^{\prime} \varphi=\delta d \varphi=0$. Among those $\psi$ for which $t \psi=t \varphi$ on $\partial \Omega, \varphi$ gives the minimum of $\|d \psi\|^{2}$.

Proof. In Proposition 3, $T=1$ and $S=0$ yield the equation $\delta d \varphi=0$. The form $\eta=\psi-\varphi$ satisfies the condition $[\eta, \varphi]=\int_{\partial \Omega} \eta \wedge * d \varphi=0$. since $t \eta=0$ on $\partial \Omega$.

An anologous result is obtained for cosemiharmonic forms:

THEOREM 7. Let $\varphi$ be a cosemiharmonic form, i.e. $\Delta^{\prime \prime} \varphi=d \delta \varphi=0$. Among those $\phi$ for which $n \psi=n \varphi$ on $\partial \Omega, \varphi$ gives the minimum of $\|\delta \phi\|^{2}$.

Proof. Take $T=0$ and $S=1$, and replace $\varphi$ by $* \varphi$ in Theorem 6 . The result follows immediately.

A coclosed harmonic form $\varphi$ satisfies $\Delta \varphi=0$ and $\delta \varphi=0$. Since the two equations lead to $\delta d \varphi=\Delta \varphi-d \delta \varphi=0$, a coclosed harmonic form is semiharmonic. Duff [3] showed that there exists a coclosed harmonic form $\varphi$ having assigned boundary values to $t \varphi$. Theorem 6 together with Duff's result gives us an interesting property of coclosed forms:

Corollary 3. Among p-forms $\phi$ with given boundary values t $\psi$, there is a form $\varphi$ minimizing the norm $\|d \psi\|$, and $\varphi$ is a coclosed harmonic form.

We remark that $\varphi$ is not unique.

Concerning the decomposition, we can state:

Corollary 4. Any form $\phi$ with $\|d \phi\|<\infty$ can be decomposed as $\phi=\varphi+\eta$, where $\varphi$ is a coclosed harmonic form with $t \varphi=t \psi$ on $\partial \Omega$ and

$$
\|d \phi\|^{2}=\|d \varphi\|^{2}+\|d \eta\|^{2}
$$

Observe that the above decomposition is not unique, but $\varphi$ is uniquely determined up to additive harmonic fields $\rho$ with $t \rho=0$; we recall that harmonic fields 
are solutions of $d \rho=\delta \rho=0$. Let $\phi=\varphi_{1}+\eta_{1}$ and $\phi=\eta_{2}+\varphi_{2}$ be two decompositions. Then $\varphi_{1}-\varphi_{2}=\eta_{2}-\eta_{1}=\rho$ is coclosed harmonic with $t \rho=0$. This means $\mathrm{d} \rho=0$ (see Duff-Spencer [4, p. 132]). Hence $\rho$ is a harmonic field.

Clearly among all $p$-forms $\phi$, the functional $\left\{\|d \psi\|^{2}+\|\delta \phi\|^{2}\right\}$ is minimized by harmonic fields and the minimum is zero. From an extremal viewpoint, the relation between forms and harmonic fields is analogous to that between $C^{1}$-functions and constant functions, which make the Dirichlet integral $D(f)$ vanish.

4. EXAmples, III. We proceed to discuss certain forms weaker than harmonic forms, viz., quasiharmonic and coquasiharmonic forms.

THEOREM 8. Let $\varphi$ be quasiharmonic, i.e., $d \Delta \varphi=0$. Among p-forms $\phi$ with $n d \psi=n d \varphi$ on $\partial \Omega$, $\varphi$ gives the minimum of $\left\|\Delta^{\prime} \phi\right\|=\|\delta d \psi\|$.

Proof. We use Proposition 3. Take $T=0, S=d$. Then equation (11) reduces to $d \delta d \varphi=0$, that is, $d \Delta \varphi=0$. The form $\eta=\psi-\varphi$ satisfies

$$
[\eta, \varphi]=-\int_{\partial \Omega} \delta d \varphi \wedge * d \eta
$$

This vanishes since $t * d \eta=* n d \eta=0$. The conclusion follows by Proposition 3 .

Next we take $T=\delta, S=0$, and obtain:

Theorem 9. Let $\varphi$ be coquasiharmonic, i.e., $\delta \Delta \varphi=0$. Among p-forms $\phi$ with $t \delta \psi=t \delta \varphi$ on $\partial \Omega, \varphi$ gives the minimum of $\left\|\Delta^{\prime \prime} \phi\right\|$.

We omit the proof since it is analogous to that of Theorem 8.

§ 3. Extremal properties of harmonic, $\boldsymbol{P}$-harmonic, quasiharmonic and $\boldsymbol{P}$ quasiharmonic functions.

1. Since functions are forms of degree 0 , the previous discussion is applicable to functions as a special case.

In this section we include several results for functions, some of which are classical, in order to stress that the underlying idea in the minimum problem of both forms and functions comes from the simple basic formulas in $\S 1$. 1 .

2. We state Proposition 1 in terms of functions:

Proposition 4. Let $T$ and $P$ be as in $\S 1.2$. If a function $u$ satisfies

$$
\delta d T u+P T u=0,
$$

then among functions $v$ with $t T v=t T u$ on $\partial \Omega$, $u$ gives the minimum of $\left\{\|d T v\|^{2}\right.$ $+(P T v, T v)\}$. Here $v$ is assumed to be sufficiently smooth so that dTv is well-defined.

We apply this to special cases of $T$ and $P$. First we take $T=1$. Then equation 
(13) reduces to $\Delta u+P u=0$, where

$$
\Delta \equiv-\frac{1}{\sqrt{ } g} \sum_{\imath} \frac{\partial}{\partial x^{2}}\left(\sum_{\jmath} \sqrt{g} g^{\imath \jmath} \frac{\partial}{\partial x^{2}}\right)
$$

Here $\left(g^{2 j}\right)$ is the inverse of the metric tensor $\left(g_{i j}\right)$, and $g$ is the determinant of $\left(g_{i j}\right)$.

A solution of $\Delta u+P u=0$ is a P-harmonic function, and the functional $\|d v\|^{2}+(P v, v)$ is the energy integral $E(v)$.

From Proposition 4, we have immediately:

THEOREM 10. A P-harmonic function $u$ minimizes the energy integral $E(v)$ among $v \in C^{1}$ with $v=u$ on $\partial \Omega$.

Since the Dirichlet problem for $\Delta u+P u=0$ is known to have a unique solution we obtain the following orthogonal decomposition (cf., e.g., Garabedian [5, p. 276]):

Corollary 5. Any $v \in C^{1}(\Omega) \cap C(\bar{\Omega})$ has the decomposition $v=u+h$, where $u$ is $P$-harmonic with $u=v$ on $\partial \Omega, h$ is in $C^{1}(\Omega)$, and $E(v)=E(u)+E(h)$.

In the case $P \equiv 0$ we have a classical theorem on harmonic functions:

Corollary 6. A harmonic function $u$ minimizes $D(v)$ among $v \in C^{1}(\Omega)$ with $v=u$ on $\partial \Omega$. Explicitly, $D(v)=D(u)+D(h)$, where $h=v-u$.

Furthermore:

Corollary 7. Any $v \in C^{1}(\Omega) \cap C(\bar{\Omega})$ has the orthogonal decomposition $v=u+h$, where $u$ is harmonic with $v=u$ on $\partial \Omega$.

3. Next we take $T=* d$. Then equation (11) is $\delta d * d u+P * d u=0$, that is, $d \delta d u+P d u=0$. Therefore $d \Delta u+P d u=0$; we call a solution $u P$-quasiharmonic.

THEOREM 11. A P-quasiharmonic function $u$ minimizes the functional $\left\{\int_{\Omega}(\Delta v)^{2} d V+\int_{\Omega} P|\operatorname{grad} v|^{2} d V\right\}$ among $v \in C^{2}(\bar{\Omega})$ with $\partial v / \partial n=\partial u / \partial n$ on $\partial \Omega$.

Proof. Proposition 4 shows that $u$ minimizes $\|d * d v\|^{2}+(P d v, d v)$ among $v$ with $t * d v=t * d u$ on $\partial \Omega$. But

$$
\begin{aligned}
& \|d * d v\|^{2}=\|* d * d v\|^{2}=\|\delta d v\|^{2}=\|\Delta v\|^{2}=\int_{\Omega}(\Delta v)^{2} d V \\
& (P d v, d v)=\int_{\Omega} P d v \wedge * d v=\int_{\Omega} P|\operatorname{grad} v|^{2} d V
\end{aligned}
$$

Here $|\operatorname{grad} v|^{2}=\sum \mathrm{g}^{\imath j}\left(\partial v / \partial x^{i}\right)\left(\partial v / \partial x^{j}\right)$, and $d V$ is the volume element.

Finally, note that the boundary condition leads to $\partial v / \partial n=\partial u / \partial n$. This completes the proof.

In the case $P \equiv 0$, equation (11) is $\Delta u=$ const. We call its solutions quasiharmonic functions, 
Corollary 8. A quasiharmonic function u minimizes the integral $\int_{\Omega}(\Delta v)^{2} d V$ among $v \in C^{2}$ with $\partial v / \partial n=\partial n / \partial n$ on $\partial \Omega$.

We can show that the Neumann problem for $d \Delta u=0$ has a solution, unique up to additive constants. From this the quasiharmonic decomposition follows.

Corollary 9. Any $C^{2}$-function $v$ has the decomposition $v=u+h$, where $u$ is quasiharmonic with $\partial u / \partial n=\partial v / \partial n$ on $\partial \Omega$, and $\|\Delta v\|^{2}=\|\Delta u\|^{2}+\|\Delta h\|^{2}$.

\section{REFERENCES}

[1] Duff, G. F. D., Boundary value problems associated with the tensor Laplace equation Canad. J. Math. 5 (1953), 57-80.

[2] Duff, G. F. D., A tensor boundary value problem of mixed type. Ibid. 6 (1954), $427-440$.

[3] DufF, G. F. D., Various classes of harmonic forms. Algebraic geometry and topology, A symposium in honor of S. Lefschetz. Princeton Unıv. Press, 1957, 129-138.

[4] Duff, G. F. D., And D. C. Spencer, Harmonic tensors on Riemannian manifolds with boundary. Ann. of Math. 56 (1952), 128-155.

[5] Garabedian, P. R., Partial differential equations. Wiley, New York, 1964, 672 pp.

Department of Mathematics,

UNIVERSITY OF CALIFORNIA,

Los ANGeles, U.S.A. 